\title{
The Growing Burden of Major Depressive Disorders (MDD): Implications for Researchers and Policy Makers
}

\author{
David Proudman ${ }^{1} \cdot$ Paul Greenberg ${ }^{2} \cdot$ Dave Nellesen $^{1}$
}

Accepted: 3 May 2021 / Published online: 20 May 2021

(c) The Author(s) 2021

Major Depressive Disorder (MDD) is one of the most burdensome illnesses globally, with significant negative impacts on activities of daily living, quality of life, cognitive function, and employment status and work productivity [1,2]. Researchers studying MDD are challenged by the complex nature of the disease, which is often comorbid with other chronic and acute conditions, including both physical and psychiatric disorders. In particular, the treatment pathway for patients with MDD is remarkably complicated, with extensive variability in disease presentation, uncertainty of diagnoses, and heterogeneity of treatment effects.

The research featured in this special issue of PharmacoEconomics draws on findings from a variety of countries across North America, Europe, Asia, and Oceania. Although differences in culture as well as health and social care systems complicate research in MDD, a broad survey of studies of the burden of MDD provides an opportunity for new insights concerning the many determinants of health outcomes associated with this disease.

This editorial overview surveys these studies, focusing on several unifying themes:

1. Growing prevalence of MDD, particularly during the coronavirus disease 2019 (COVID-19) pandemic

2. Research concerning the cost of MDD

3. Economic burden of MDD in subpopulations

4. Advances in analytical methods

5. Implications for researchers and policy makers.

Dave Nellesen

dave.nellesen@analysisgroup.com

1 Analysis Group, Menlo Park, CA, USA

2 Analysis Group, Boston, MA, USA

\section{Growing Prevalence of MDD}

The prevalence of MDD is extraordinarily high and growing over time; between 2005 and 2018, the number of adults in the USA diagnosed with MDD increased from 13.7 million to 17.5 million, with the prevalence rate rising from 6.8 to $7.1 \%[3,4]$. The rising prevalence is unevenly distributed by age, with the greatest increase seen among 18- to 34-yearolds [4].

With the severe impact of the COVID-19 pandemic on mental health, much new research and analysis will be needed to assess the growing burden of MDD. Starting in early 2020, the pandemic resulted in new, stressful, and at times overwhelming worries about the health effects of the virus itself, heightened concerns about potential loss of employment, and prolonged social isolation involving greatly reduced interaction with family and friends. Actions taken to reduce the risk of COVID transmission, such as quarantining and social distancing, may have intensified feelings of loneliness, anxiety, and depression for many people. Recent data from the Centers for Disease Control and Prevention (CDC) show that the US prevalence of MDD has increased substantially from $7 \%$ prior to the pandemic to $27 \%$ during the first year of the pandemic, and MDD with anxiety disorders has increased from 11 to $38 \%[5,6]$ (see Fig. 1).

Such a sudden and unprecedented increase in the prevalence of MDD has certainly added to the global burden of disease, with the younger age cohorts meriting particular research attention. In addition, the magnitude and differences in effects across geographies and healthcare systems remain uncertain and will also likely be the subject of much future pharmacoeconomic research. 


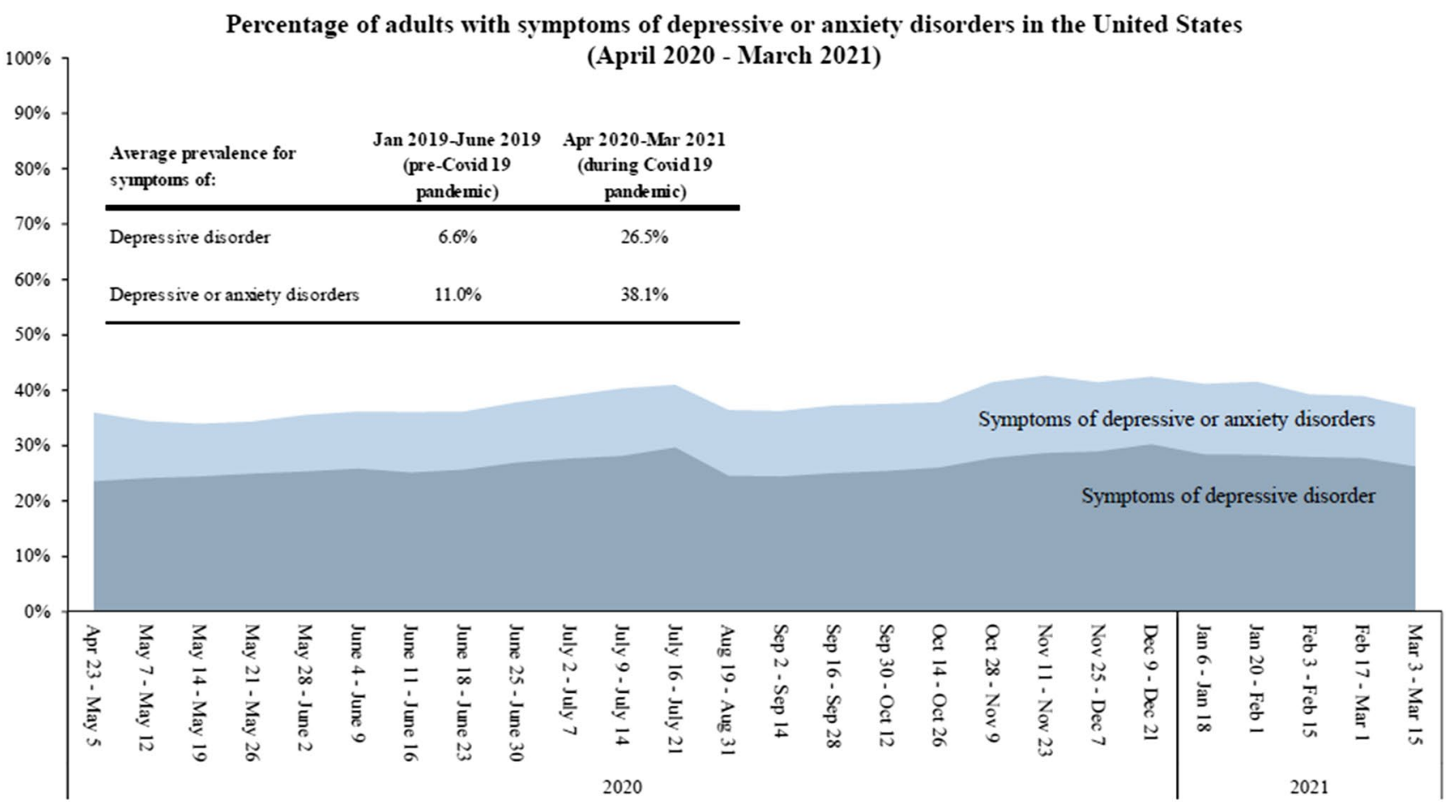

Source: The Centers for Disease Control and Prevention, available at https://data.cdc.gov/NCHS/Indicators-of-Anxiety-or-Depression-Based-on-Repor/8pt5-q6wp. Accessed 3/30/2021.

Notes:

[1] Prevalence data from 7/23/2020 through 8/17/2020 and from 12/23/2020 through 1/4/2021 were unavailable.

[2] The term major depressive disorder (MDD) is used in the ensuing article "The Economic Burden of Adults with Major Depressive Disorder in the United States (2010 and 2018)" of this special issue where data from the National Survey on Drug Use and Health (NSDUH) were analyzed. The prevalence of MDD in 2018 based on NSDUH data was estimated at 7.1\%

Fig. 1 Weekly prevalence of major depressive disorders, April 2020-March 2021

\section{Research Concerning the Cost of MDD}

Cost of illness is an essential consideration underlying the efficient allocation of economic resources. Such studies can illuminate cost drivers as well as patient subgroups associated with high costs, provide information necessary to rationalize health system improvements, and inform design and development of cost-effective new health technologies.

It is difficult to undertake direct comparisons across countries given large variation in medical cost estimates, due to a range of differences including insurance availability, treatment rates, pricing of treatments, and overall prevalence of disease. Nonetheless, relative patterns can generate insights and inform hypotheses that are relevant in comparing outcomes in one country with those in another.

Greenberg et al. estimate that costs of adults with MDD in the USA rose from US $\$ 236.6$ billion in 2010 to US $\$ 326.2$ billion in 2018 [4]. Approximately $40 \%$ of the rise in economic burden is attributable to the increase in prevalence of disease over this period, while the remaining $60 \%$ is due to increased costs per person, especially rising absenteeism and presenteeism costs in the workplace [4]. An analysis by König et al., based on the German Health Interview and Examination Survey for Adults, finds that excess costs of depression per person with MDD amount to $€ 5047$, with direct costs accounting for $€ 2212$ of the total in 2011 [7]. Kan et al. estimate the mean cost of a treatment episode in the Netherlands for MDD patients in a specialist mental healthcare setting to be $€ 21,186$ (demonstrating a large skew compared to the median in the same population of €2320) [8]. In Australia, Callander et al. estimate the total cost of services accessed from birth to 12 months postpartum by women with postpartum MDD to be A $\$ 14,090,636 \%$ higher than women without postpartum MDD after accounting for differences in private hospital use, delivery type, clinical characteristics, and socioeconomic status (SES) [9]. These findings underscore that small groups of MDD patients can meaningfully affect overall costs [8].

Drug costs are an important subset of direct medical costs. Greenberg et al. estimate that US pharmaceutical expenditures decreased from US $\$ 31.6$ billion in $2010(13 \%$ of direct costs) to US\$20.4 billion in 2018 (6\% of direct costs) [4]. This equates to approximately US $\$ 2034$ per patient with MDD in 2010 and US\$1164 in 2018 [4]. The 
authors suggest that this decline in costs is largely driven by the increasing reliance on generic prescription antidepressants over time in the USA [4].

A decline in the cost of pharmaceutical treatments for MDD from a payer and patient perspective may suggest an increase in the value of healthcare delivered. However, it may also reflect limited entry of new and improved (more costly) pharmaceutical treatments. Among numerous obstacles, the cost of clinical trials has risen over time; it takes $35 \%$ longer to bring central nervous system (CNS) drugs to market compared with drugs in other disease areas, and markedly fewer of the clinical candidates in this area reach the market [10]. According to the UK trade group the ABPI, $38 \%$ of global R\&D is invested in oncology, whereas only $7 \%$ is invested in CNS diseases [11]. Given the unmet need for more effective treatments, together with the extraordinary increase in MDD prevalence during the pandemic, additional incentives for pharmaceutical innovation may be needed to spur innovation in this therapeutic area.

\subsection{Cost of Suicide}

Greenberg et al. estimate suicide-related costs totaling US $\$ 13.4$ billion, or $4 \%$ of the total economic burden of adults with MDD [4]. Moreover, Zhdanava et al. note that costs incurred after a suicide attempt trend higher compared with those before the attempt, and that the majority of these patients receive care in high-cost inpatient or emergency room (ER) settings [12]. This suggests that early diagnosis and intervention to address suicidal behavior might improve economic as well as clinical outcomes [12].

\subsection{Indirect Cost of MDD}

A large percentage of the economic burden of adults with MDD stems from workplace-related costs that result from either missed time from work (absenteeism) or reduced productivity while at work (presenteeism). Greenberg et al. estimate that for every dollar spent on direct costs of MDD, an additional US\$2.30 is incurred in MDD-related workplace costs and suicide-related costs, and another US $\$ 3.49$ is incurred in workplace costs related to comorbid conditions [4].

\section{Economic Burden of MDD in Subpopulations}

A consistent trend over the past 2 decades in the analysis of health economic and outcomes data has been an increased focus on various key patient subgroups, highlighting the heterogeneity of patient experiences with MDD. For example, the number of published articles focusing on MDD and suicidal ideation grew by 2180 (234\%) between 2005 and 2019. Similarly, articles on MDD and cancer increased by 3841 (108\%) over that same time period. Increased research attention has also focused on MDD by comorbid conditions (e.g., chronic pain), age cohort, and depression types (e.g., treatment-resistant depression [TRD], postpartum depression [PPD]) (see Fig. 2).

\subsection{Comorbid Conditions}

Major Depressive Disorder (MDD) that is comorbid with other physical or psychiatric diagnoses often results in more complex patient experiences with treatment and increased healthcare resource utilization. Therefore, a complete understanding of the economic burden of adults with MDD should include particular attention to various patient subgroups with these characteristics in mind.

Kan et al. show that the presence of psychiatric comorbidities, such as bipolar or schizophrenia, is associated with higher treatment costs as well as more complex disease management programs, compared with those without psychiatric comorbidities [8]. For instance, patients with MDD and comorbid personality disorder incur total cost per treatment of $€ 23,933$, whereas patients with MDD alone incur total cost per treatment of $€ 11,612$ [8]. Yim et al. find that cancer patients tend to have more frequent general practitioner visits for anxiety and depression than those without cancer, resulting in higher overall healthcare costs [13]. Similarly, Shah et al. estimate that of the excess cost burden of MDD patients with TRD compared to MDD patients without TRD, $11.2 \%$ is explained by the higher number of chronic noncancer pain conditions and $8.2 \%$ by the increased prevalence of sleep and anxiety disorders in TRD patients [14].

\subsection{Age Comparisons}

Over the past decade, the proportion of MDD attributable to younger people has grown. Studies by König et al. and Greenberg et al. report that these age groups have higher excess costs compared with older age groups [4, 7]. König et al. report that excess medical costs range from $€ 4819$ for 30- to 44-year-olds to $€ 1242$ for 45 - to 64-year-olds [7]. Greenberg et al. estimate that in the USA, adults with MDD aged $18-25$ years had a $73.7 \%$ increase in total incremental direct costs between 2010 and 2018, while the costs for those aged $35+$ years decreased by $16.5 \%$ over this period [4].

\subsection{Treatment-Resistant Depression and Postpartum Depression}

Patients with more severe forms of MDD generally incur more healthcare costs and experience poorer health outcomes. One prominent subgroup within MDD is 


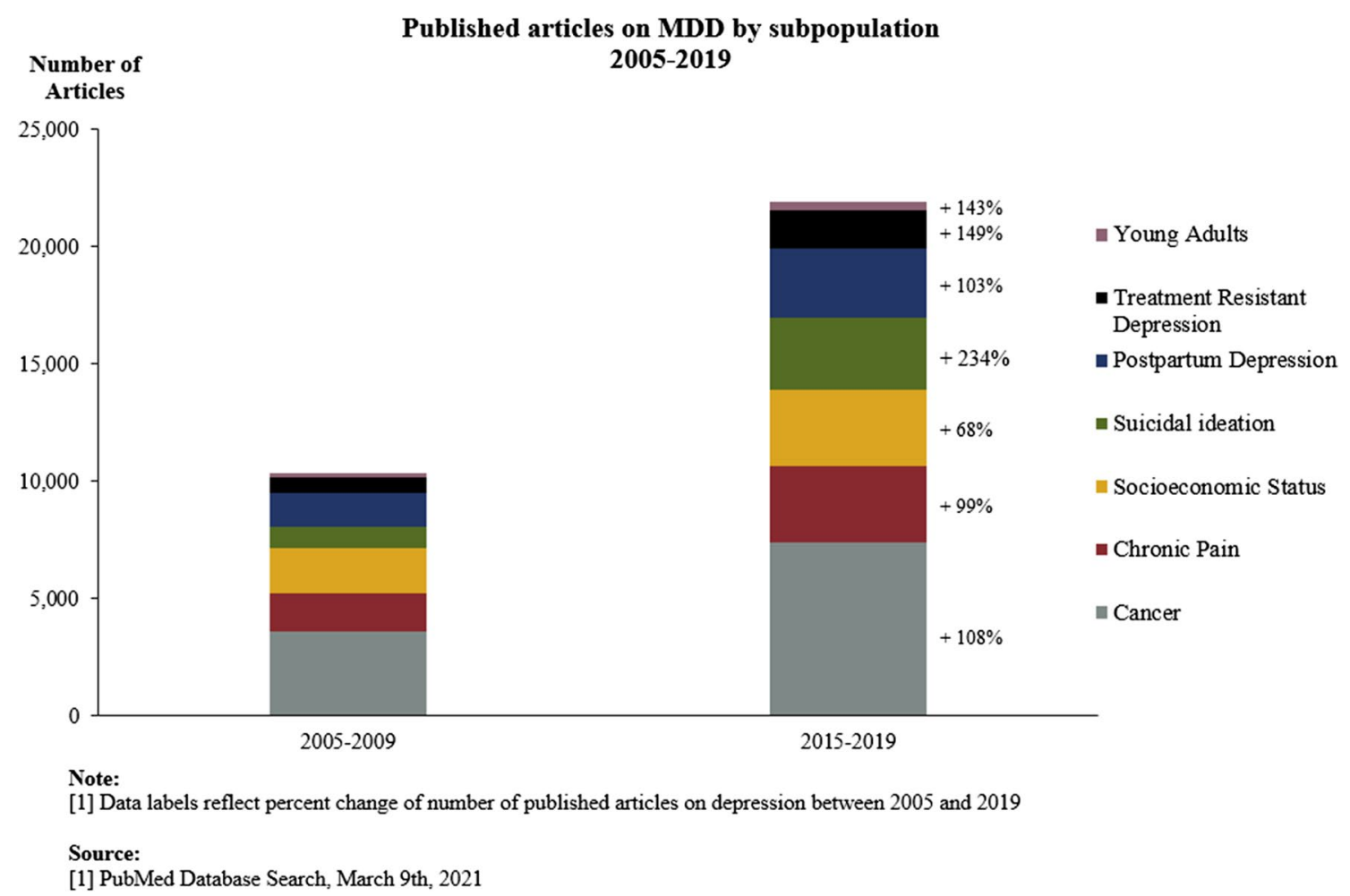

Fig. 2 Growth in research on areas of high unmet need in major depressive disorders (MDD)

characterized by TRD. This condition is associated with high unmet need following administration of treatment. Adults with TRD often have longer episodes of MDD and greater symptom severity, leading to worse clinical outcomes and higher economic costs [14]. Shah et al. estimate that patients with TRD have more inpatient stays (incidence rate ratio $[\mathrm{IRR}]=1.30)$ and $\mathrm{ED}$ visits $(\mathrm{IRR}=1.21)$ as well as higher levels of other MDD-related healthcare utilization compared with non-TRD patients in the USA [14]. They also estimate that prescription drugs make up the highest proportion of excess costs for US patients with TRD, as they often try many different treatment options before achieving remission [14].

In general, TRD patients have extremely high rates of distress and poor functioning [14]. Arnaud et al. estimate that delays in achieving treatment stability are associated with increased direct and indirect costs [15]. Commercially insured patients in the USA who achieve treatment stability after one line of pharmacotherapy are estimated to incur US\$9975 in medical costs, but those who stabilize after three or more lines incur medical costs of US\$21,259 in the first 12 months following initiation of antidepressants [15]. They also note that the current standard of care, which involves sequential step pharmacotherapy, can lead to high costs as patients may repeatedly switch treatments if symptoms do not improve [15].
Another form of illness that garners a lot of research attention is PPD. Callander et al. show that PPD is found in a higher percentage of women with pre-existing mental health conditions and results in sizable increases in healthcare utilization [9].

\subsection{Socioeconomic Status}

Several studies examine the effect of SES on depressionrelated costs and offer different insights depending on local conditions. Callander et al. find that among Australian women with PPD, those with lower SES had lower total healthcare costs than women with higher SES, most likely as a result of differential access to care [9]. Those with lower SES have fewer inpatient episodes, specialist consultations, and prescriptions but higher general practitioner consultations and emergency department presentations [9]. In Germany, König et al. find that direct medical costs tend to be lower among MDD patients with higher SES. The authors hypothesize that this is likely to be due to higher SES being associated with lower incidence of mental illness. In addition, those with higher SES often seek out more targeted care, resulting in less overall healthcare utilization [7]. The authors also find that having a migrant background is associated with lower healthcare costs due to little access to care [7]. As with many other diseases, SES has been shown to 
greatly affect access to care, insurance status, consistency in treatment follow-up, and other factors that affect the clinical outcomes of patients with MDD. These factors all contribute to the heterogeneity and, ultimately, the economic burden of disease and therefore can be critical inputs in economic models [16].

\section{Advances in Analytical Methods}

Studies included in this special issue on MDD draw on advanced analytical methods to address a variety of important research questions. Higher quality data sources are fundamental to improving our understanding of the economics of MDD, while advances in statistical analyses and patientcentered outcomes enable more accurate and granular capture of the true burden of disease. In addition, new methods that encompass multiple stakeholders allow for consideration of diverse perspectives in the assessment of the value of new interventions to treat MDD.

Zhdanava et al. conduct a retrospective longitudinal study for the period 2014-2019 using the IBM MarketScan Commercial and Medicare Supplemental Databases to identify patients with MDD and acute suicidal ideation or behavior [12]. The authors perform iterative hierarchical clustering, a machine-learning technique that identifies and combines individuals with similar characteristics into distinct groupings. Before the advent of this data-driven methodology, most authors attempted to cluster patients by anchoring on clinical consensus. In contrast, this clustering methodology is unsupervised (i.e., not informed by inputs such as clinical assumptions), and can identify previously unknown patterns in population characteristics. In this study, new insights are developed regarding the patterns of exposure to the healthcare system prior to a suicide-related event, a technique that holds promise to elucidate additional patient subgroups and treatment strategies in MDD.

König et al. estimate annual excess costs per patient in 2011 by comparing survey data for those who did and did not self-report symptoms of MDD [7]. Differences in characteristics between depressed and non-depressed participants are adjusted using entropy balancing, with attention to sociodemographic and clinical covariates. Entropy balancing is a reweighting method that, in many circumstances, has shown to be a superior covariate balancing technique when compared with traditional propensity score weighting. It has also been used in excess cost studies for many other disease areas. This approach can strengthen various types of analysis in MDD economic research, including observational cohort designs, survey analyses, and generalized linear models, since the approach enables numerous covariates to be adjusted simultaneously.
In addition to the application of advanced analytical approaches, the refinement of methodological practices may lead to more accurate findings and outcomes, as demonstrated in the capture of quality-of-life measurements. Hays and Fayers conduct a review of studies related to healthrelated quality of life (HRQoL) metrics and show that there is an overlap between stand-alone depressive symptom measures and many HRQoL instruments [17]. The authors note the importance of this overlap for those who are designing trials and surveys or using the resulting data to inform clinical and economic assessment. Ignoring this overlap between clinical and quality-of-life metrics can lead to double-counting of depression characteristics and to potentially misleading conclusions about the prognosis of disease for resource utilization, mortality, and other key outcomes.

Xie et al. develop an iterative multi-stakeholder model development approach, which includes an advisory group that incorporates heterogeneous perspectives [16]. Compared with traditional approaches, engagement with key stakeholders offers promise in building better inputs from underrepresented stakeholder groups such as patients or employers, validating key assumptions, and identifying methodological challenges early on. Additionally, it may improve the identification and incorporation of relevant data inputs and help foster additional research and data collection efforts to improve the quality and relevance of value assessments. This is a challenging task given the difficulty of achieving agreement and balance among many stakeholders, but one that warrants further attention in MDD as well as other areas of mental health treatment.

\section{Implications for Researchers and Policy Makers}

The global economic burden of all mental health conditions is estimated to rise from US\$2.5 trillion in 2010 to US\$6 trillion by 2030 [18]. MDD is a prominent contributor to this overall burden, as it affects a large proportion of the global population, has a major adverse impact on those affected, and imposes immense costs on insurers, employers, patients, and caregivers. While the impact of the COVID-19 pandemic is undoubtedly enormous, its long-term effects remain uncertain. But it is already clear that young adults are particularly vulnerable to the volatility of the current environment, and will likely be a substantial contributor to the expected rise in the global burden of MDD. As MDD trends younger, the lifetime costs of illness could well increase unless substantial inroads are made in successfully treating its sufferers with innovative forms of outreach and intervention.

Given that MDD patients with low SES tend to have higher prevalence of mental illness, innovative social 
policies, such as family strengthening programs, housing vouchers, or increased funding (e.g., through COVID-relief legislation in the USA), may succeed in extending healthcare coverage and access to underserved populations with MDD while increasing health equity and improving health outcomes. While direct costs may increase as a result of greater health service utilization, some accompanying cost offsets from reduced absenteeism or presenteeism could be realized at the same time.

Where not already used for MDD, payment models that focus on reimbursement methods like bundled payments for episodes of care, which would require risk adjustment to account for each specific beneficiary mix, could be beneficial [14]. Episode-of-care reimbursement policies can encourage healthcare systems to use more integrated care and optimize treatment according to patient characteristics. In such a reimbursement model, risk adjustment factors can be improved with an understanding of sub-populations that incorporate attention to the patterns of care for different patient types. For example, family and social support, family history of mental illness, substance abuse, medication adherence, symptom severity, recorded suicide attempts, and age of onset are all factors associated with prognoses related to patients with MDD that could be used in refined reimbursement models.

Research presented in this special issue shows how resource use, cost, and clinical outcomes vary widely among subgroups of MDD patients, highlighting opportunities for researchers and policy makers to identify, characterize, and address the needs of key subpopulations more effectively.

Acknowledgements The authors would like to thank Cassie Regan, a full time employee of Analysis Group, for research and editorial assistance in preparing this article. Support for this article was provided by Analysis Group, Inc. The authors have no conflicts of interest that are directly relevant to the content of this article.

\section{Declarations}

Conflict of interest The authors (DP, PG, DN) report the following details of affiliation or involvement in an organization or entity with a financial or non-financial interest in the subject matter or materials discussed in this article: Employment, Analysis Group (a healthcare consulting firm).

Open Access This article is licensed under a Creative Commons Attribution-NonCommercial 4.0 International License, which permits any non-commercial use, sharing, adaptation, distribution and reproduction in any medium or format, as long as you give appropriate credit to the original author(s) and the source, provide a link to the Creative Commons licence, and indicate if changes were made. The images or other third party material in this article are included in the article's Creative Commons licence, unless indicated otherwise in a credit line to the material. If material is not included in the article's Creative Commons licence and your intended use is not permitted by statutory regulation or exceeds the permitted use, you will need to obtain permission directly from the copyright holder. To view a copy of this licence, visit http://creativecommons.org/licenses/by-nc/4.0/.

\section{References}

1. James SL, Abate D, Abate KH, et al. Global, regional, and national incidence, prevalence, and years lived with disability for 354 diseases and injuries for 195 countries and territories, 1990-2017: a systematic analysis for the Global Burden of Disease Study 2017. Lancet. 2018:392(10159):1789-858.

2. Clark M, DiBenedetti D, Perez V. Cognitive dysfunction and work productivity in major depressive disorder. Expert Rev Pharmacoecon Outcomes Res. 2016;16(4):455-63.

3. Greenberg PE, Fournier AA, Sisitsky T, Pike CT, Kessler RC. The economic burden of adults with major depressive disorder in the United States (2005 and 2010). J Clin Psychiatry. 2015;76(2):155-62.

4. Greenberg P, Fournier A-A, Sisitsky T, et al. The economic burden of adults with major depressive disorder in the United States (2010 and 2018). Pharmacoeconomics. 2021. https://doi.org/10. 1007/s40273-021-01019-4.

5. Anxiety and Depression. 2021. https://www.cdc.gov/nchs/covid 19/pulse/mental-health.htm. Accessed 4 March 2021.

6. Centers for Disease Control and Prevention. Early release of selected mental health estimates based on data from the JanuaryJune 2019 National Health Interview Survey. Atlanta: Centers for Disease Control and Prevention; 2020.

7. König H, Rommel A, Thom J, et al. The excess costs of depression and the influence of sociodemographic and socioeconomic factors: results from the German Health Interview and Examination Survey for Adults (DEGS). Pharmacoeconomics. 2021. https:// doi.org/10.1007/s40273-021-01000-1.

8. Kan K, Lokkerbol J, Jörg F, Visser E, Schoevers R, Feenstra T. Real-world treatment costs and care utilization in patients with major depressive disorder with and without psychiatric comorbidities in specialist mental healthcare. Pharmacoeconomics. 2021. https://doi.org/10.1007/s40273-021-01012-x.

9. Callander E, Gamble J, Creedy D. Postnatal major depressive disorder in Australia: Inequalities and costs of healthcare to individuals, governments and insurers. Pharmacoeconomics. 2021. https:// doi.org/10.1007/s40273-021-01013-w.

10. Kaitin KM, C. A dearth of new methods. 2011. https://www.scien tificamerican.com/article/a-dearth-of-new-meds/. Accessed 16 Mar 2021.

11. Massaro TJ, Chen Y, Ke Z. Efficacy and safety of thrombopoietin receptor agonists in children with chronic immune thrombocytopenic purpura: meta-analysis. Platelets. 2019;30:1-8.

12. Zhdanava M, Voelker J, Pilon D, et al. Cluster analysis of care pathways in adults with major depressive disorder with acute suicidal ideation or behavior in the United States. Pharmacoeconomics. 2021. https://doi.org/10.1007/s40273-021-01042-5.

13. Yim J, Shaw J, Viney R, Arora S, Ezendam N, Pearce A. Investigating the impact of comorbid anxiety and depression on health service use in cancer survivors. Pharmacoeconomics. 2021. https://doi.org/10.1007/s40273-021-01016-7.

14. Shah D, Allen L, Zheng W, et al. Economic burden of treatmentresistant depression among adults with chronic non-cancer pain conditions and major depressive disorder. Pharmacoeconomics. 2021. https://doi.org/10.1007/s40273-021-01029-2.

15. Arnaud A, Suthoff E, Tavares R, Zhang X, Ravindranath A. The increasing economic burden with additional steps of pharmacotherapy in major depressive disorder. Pharmacoeconomics. 2021. https://doi.org/10.1007/s40273-021-01021-w. 
16. Xie R, deFur Malik E, Linthicum M, Bright J. Putting stakeholder engagement at the center of health economic modeling for health technology assessment in the United States. Pharmacoeconomics. 2021. https://doi.org/10.1007/s40273-021-01036-3.
17. Hays R, Fayers P. Overlap of depressive symptoms with healthrelated quality-of-life measures. Pharmacoeconomics. 2020. https://doi.org/10.1007/s40273-020-00972-w.

18. Health TLG. Mental health matters. Lancet Global Health. 2020;8(11):e1352. 